\title{
Das Schädel-Hirn-Trauma im Säuglings- und Kindesalter
}

Angela Brentrup, Stephanie Schipmann

\section{Definition}

Das Schädel-Hirn-Trauma (SHT) ist definiert als die Folge einer Gewalteinwirkung, die zu einer Verletzung und/ oder Funktionsstörung des Gehirns geführt hat [1]. Die Verletzungen können dabei den knöchernen Schädel, die Kopfschwarte, Hirngewebe und auch Hirngefäße betreffen. In Abhängigkeit vom Verletzungsgrad der Weichteile, des Knochens und der Dura mater wird zwischen einem offenen (Verbindung des Schädelinneren nach außen durch Perforation von Haut, Knochen und Dura) und einem gedeckten SHT unterschieden.

Inzidenz, Unfallmechanismus, Ursache, Diagnostik, Therapie und Langzeitverlauf unterscheiden sich bei Schädel-Hirn-Traumata allein durch das bestehende Lebensalter zum Unfallzeitpunkt.

\section{Epidemiologie}

Schädel-Hirn-Traumata stellen ein signifikantes medizinisches, soziales und ökonomisches Problem dar. Sie sind bis heute einer der häufigsten Gründe für Behinderungen und Todesfälle im Kindes- und Jugendalter. Insgesamt betreffen 29,7\% aller Schädel-Hirn-Traumata Kinder unter 16 Jahren [2]. Eine in Deutschland 2014 vom Statistischen Bundesamt veröffentliche Studie zeigte, dass innerhalb eines Jahres 581 von 100000 Kindern zwischen 1 und 15 Jahren mit einer Schädel-Hirn-Verletzung stationär behandelt werden; über $90 \%$ davon mit einer Gehirnerschütterung. Weniger als $10 \%$ sind als mittelschweres oder schweres SHT einzustufen.

Kinder unter 6 Jahren weisen eine deutlich höhere Inzidenz mit 721 Fällen pro 100000 auf [2]. Die Sterblichkeit beträgt insgesamt ca. 0,5\%, bei schwerem SHT jedoch bis zu 14\%. Bei den Jugendlichen und jungen Erwachsenen liegen die Hospitalisierungsraten deutlich unter den Werten der Säuglinge und Kleinkinder. Die Kopfverletzungen nehmen auch hier den höchsten Rang ein, der Anteil ist aber deutlich geringer als bei den Vergleichsgruppen der unter 5-Jährigen. [3].

In den vergangenen Jahren ist ein deutlicher Abwärtstrend der Verletzungen mit Todesfolge zu beobachten. Ab dem 1. Lebensjahr bleibt die Kopfverletzung jedoch weiterhin die häufigste Todesursache bei Kindern und Ju- gendlichen und liegt bei 2,2 je 100000 Einwohner. Im europäischen Vergleich liegt Deutschland in Bezug auf die verletzungsbedingte Mortalität im unteren Drittel [3].

Eine Sondergruppe bilden die gewaltbedingten Todesfälle. Deren Anzahl bleibt auch im Langzeitverlauf konstant. Insbesondere sind hier die Säuglinge mit einer Rate an gewaltbedingten Verletzungen von 42 je 100000 betroffen, diese haben ein 10-fach höheres Risiko als die Altersgruppe der 10-15-Jährigen mit 4 Fällen je 100000 [3]. Die führende Ursache bei den gewaltbedingten Todesfällen sind Schädel-Hirn-Verletzungen. Hierbei steht insbesondere das Schütteltrauma im Fokus mit insgesamt ca. 100-200 Fällen/Jahr in Deutschland, welches mit einer Letalität von bis zu 30\% und dem Risiko der Entwicklung von Langzeitschäden bis zu 70\% einhergeht [4].

\section{Zusammenfassung}

Die Inzidenz der tödlichen Kopfverletzungen - bis auf die der gewaltbedingten - hat in Deutschland abgenommen. Dennoch gehörten Schädel-Hirn-Verletzungen noch zu den häufigsten Todesursachen bei Kindern zwischen 1 und 15 Jahren in Deutschland. Insbesondere in der Altersgruppe der unter 1-Jähringen ist das Schütteltrauma eine führende Ursache für Schädel-Hirn-Verletzungen.

\section{Pathophysiologie}

Bei der Schädigung des Gehirns kann zwischen primären und sekundären Verletzungen unterschieden werden:

1. Primäre Verletzungen sind sofortige Schädigungen, die durch mechanische Kräfte verursacht werden, die auf das Hirnparenchym übertragen werden und sich in Form von Kontusionsblutungen, Hämatomen, Scherverletzungen und Ödemen manifestieren. Typische einwirkende Kräfte sind direkter Aufprall, schnelle Beschleunigungen, penetrierende Verletzungen und Explosionsdruckwellen.

2. Sekundäre Verletzungen werden als Folgeschäden definiert, die sich nach Stunden und Tagen infolge veränderter Hirndurchblutung (Vasospasmen, Mikrozirkulationsstörungen) und inflammatorischen Prozessen entwickeln. Folgen sind Hypoxie, Veränderungen von Elektrolyt- und Zellmetabolismus, neuronaler Zelltod sowie die Entstehung von zerebralen Ödemen und in der Folge Anstieg des intrazerebralen Druckes [5]. 
Merke

Die anfängliche Stabilisierung des verletzten Kindes in der präklinischen und notfallmedizinischen Versorgung konzentriert sich auf die Minimierung der Folgeerscheinungen der primären Verletzungen.

\section{Ursachen}

Insbesondere im Hinblick auf die Entwicklung effektiver Präventionsstrategien kommt der Unfallursachenforschung eine besondere Bedeutung zu.

Zunächst sind aber die körperlichen Besonderheiten bez. eines Unfallhergangs bei einem Kind zu bedenken. Die Körper-Kopf-Proportionen und die Ausprägung der Schutzreflexe spielen bez. der Beteiligung des Kopfes bei einem Trauma eine entscheidende Rolle: Das Gewicht des Kopfes im Verhältnis zum Gesamtgewicht des Körpers beträgt beim Säugling 1:3 und beim Erwachsenen 1:30. Auch setzen die Schutzreflexe bei Kindern später ein, so ist die Reaktionszeit eines 5-Jährigen etwa doppelt so lang wie die eines Erwachsenen.

Generell sind häufige Ursachen für Schädel-Hirn-Traumata im Kindesalter Stürze und Verkehrsunfälle sowie Kopfverletzungen infolge eines sich bewegenden Gegenstandes oder nicht akzidentelle Verletzungen.

Insbesondere bei den Stürzen kann eine deutliche Variabilität zwischen Verletzungsursache und Alter beobachtet werden, und Veränderungen in der kindlichen Entwicklung beeinflussen die Sturzart [6].

Untersucht man Verletzungsmechanismen in verfeinerten Altersgruppen, so fällt auf, dass sich im Säuglings- und Kleinkindalter Stürze vorrangig im häuslichen Umfeld ereignen (Stürze aus Schlafgelegenheiten, vom Wickeltisch, Treppen etc.). Mit zunehmenden Aktionsradius des Kindes (Altersgruppe 4-15 Jahre) verlagert sich der Unfallort auf Spielund Sportgelände, die Natur sowie auf die Verkehrswege [7].

\section{Zusammenfassung}

Sturzunfälle spielen bei Kindern unter 14 Jahren die größte Rolle bei Schädel-Hirn-Verletzungen und sind über $90 \%$ produktbezogen. Mit zunehmendem Aktionsradius der Kinder erweitert sich das Spektrum der Unfallorte, so auch die Anzahl der Verkehrsunfälle. Das Schütteltrauma als Verletzungsmechanismus spielt mit einem Durchschnittsalter von 4 Monaten eine besondere Rolle.

\section{Klassifikation, Symptomatik und Verletzungsformen}

\section{Klassifikation}

Die Einteilung des Schweregrads eines Schädel-HirnTraumas erfolgt nach AWMF-Leitlinien (AWMF: Arbeits-
- Tab. 1 Die Frankfurter GCS (F-GCS) in Abhängigkeit vom Alter (1-24 Monate, > 24 Monate).

\begin{tabular}{|c|c|c|}
\hline \multicolumn{3}{|c|}{ Verbale Antwort (V = „verbal“) } \\
\hline & 1-24 Monate & $>24$ Monate \\
\hline 5 & fixiert, verfolgt, erkennt, lacht & $\begin{array}{l}\text { spricht verständlich, ist } \\
\text { orientiert }\end{array}$ \\
\hline 4 & $\begin{array}{l}\text { fixiert, verfolgt inkonstant, } \\
\text { erkennt nicht sicher, lacht nicht } \\
\text { situationsbedingt }\end{array}$ & $\begin{array}{l}\text { ist verwirrt, spricht unzu- } \\
\text { sammenhängend, ist } \\
\text { desorientiert }\end{array}$ \\
\hline \multirow[t]{2}{*}{3} & $\begin{array}{l}\text { nur zeitweise erweckbar, trinkt } \\
\text { und isst nicht }\end{array}$ & $\begin{array}{l}\text { antwortet inadäquat, } \\
\text { Wortsalat }\end{array}$ \\
\hline & $\begin{array}{l}\text { Bedrohreflex (ab } 4 / 12 \mathrm{~m} \text { ) nicht } \\
\text { sicher auslösbar }\end{array}$ & \\
\hline 2 & $\begin{array}{l}\text { ist motorisch unruhig, jedoch } \\
\text { nicht erweckbar }\end{array}$ & unverständliche Laute \\
\hline 1 & $\begin{array}{l}\text { tief komatös, kein Kontakt zur } \\
\text { Umwelt, keine visuell, akustisch } \\
\text { oder sensorisch ausgelöste moto- } \\
\text { rische Reizantwort }\end{array}$ & $\begin{array}{l}\text { keine verbalen Äußerun- } \\
\text { gen }\end{array}$ \\
\hline \multicolumn{3}{|c|}{ Motorische Antwort ( $\mathrm{M}=$ „,motor“ $)$} \\
\hline 6 & \multicolumn{2}{|c|}{$\begin{array}{l}\text { greift gezielt auf Aufforderung, befolgt andere motorische Auffor- } \\
\text { derungen prompt }\end{array}$} \\
\hline 5 & \multicolumn{2}{|c|}{ gezielte Abwehr eines Schmerzreizes möglich } \\
\hline 4 & \multicolumn{2}{|c|}{ ungezielte Beugebewegungen auf Schmerzreize } \\
\hline 3 & \multicolumn{2}{|c|}{$\begin{array}{l}\text { ungezielte Beugebewegungen auf Schmerzreize an den Armen, } \\
\text { Strecktendenz an den Beinen (Dekortikationshaltung) }\end{array}$} \\
\hline 2 & \multicolumn{2}{|c|}{$\begin{array}{l}\text { Extension aller } 4 \text { Extremitäten auf Schmerzreize (Dezerebrations- } \\
\text { haltung) }\end{array}$} \\
\hline 1 & \multicolumn{2}{|c|}{ keine motorische Antwort auf Schmerzreize } \\
\hline \multicolumn{3}{|c|}{ Augenöffnen ( $E=$ „eye“) } \\
\hline 4 & \multicolumn{2}{|l|}{ spontanes Augenöffnen } \\
\hline 3 & \multicolumn{2}{|l|}{ Augenöffnen auf Anruf } \\
\hline 2 & \multicolumn{2}{|l|}{ Augenöffnen auf Schmerzreiz } \\
\hline 1 & \multicolumn{2}{|l|}{ kein Augenöffnen } \\
\hline \multicolumn{3}{|c|}{ Augensymptome (OV = „oculo-vestibular“) } \\
\hline 4 & \multicolumn{2}{|c|}{$\begin{array}{l}\text { konjugierte Augenbewegungen möglich, Lichtreaktion der Pupil- } \\
\text { len auslösbar }\end{array}$} \\
\hline 3 & \multicolumn{2}{|c|}{$\begin{array}{l}\text { Puppenaugenphänomen auslösbar, dabei konjugierte Bulbus- } \\
\text { bewegungen }\end{array}$} \\
\hline 2 & \multicolumn{2}{|c|}{$\begin{array}{l}\text { Divergenzstellung der Bulbi, besonders bei Auslösen des Puppen- } \\
\text { augenphänomens oder Kaltspülung des äußeren Gehörgangs }\end{array}$} \\
\hline 1 & \multicolumn{2}{|c|}{$\begin{array}{l}\text { Ausbleiben der Augenbewegungen hierbei keine spontanen } \\
\text { Augenbewegungen; weite, lichtstarre Pupillen }\end{array}$} \\
\hline
\end{tabular}

gemeinschaft der Wissenschaftlichen Medizinischen Fachgesellschaften) in:

- leichtes SHT

- mittelschweres SHT

- schweres SHT

Grundlage für die Klassifikation sind die Dauer und das Ausmaß der posttraumatischen Bewusstseinsstörung.

Ein wichtiges Tool zur Einteilung ist die Glasgow Coma Scale (GCS), die für Kinder (<12 Jahre) in Rücksicht auf 

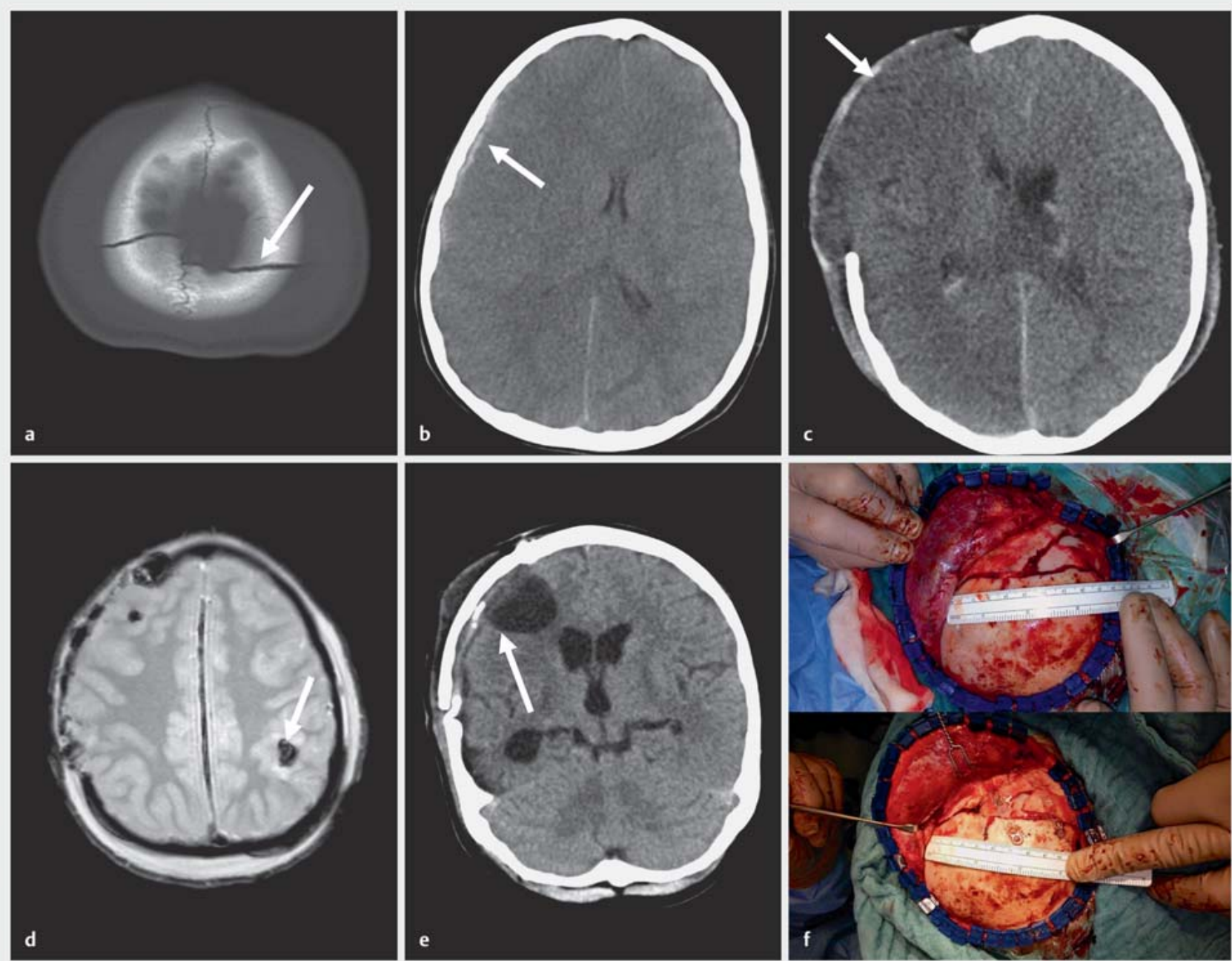

- Abb. 1 Fallbeispiel schweres Schädel-Hirn-Trauma: 6-jähriger Junge, der als Fußgänger von einem Auto erfasst wurde und ein schweres SHT mit Kalottenimpressionsfraktur (a) sowie akut subduralem Hämatom rechts (b) erlitt. Es erfolgten die dekompressive Hemikraniektomie, Hämatomausräumung und vorübergehende Anlage einer ICP-Sonde (c). In einem ergänzend durchgeführten MRT zeigt sich zudem eine Kontusionsblutung rechts parietookzipital (MRT T2hem ax) (d). Drei Monate später konnte die Kalotte, die in der Zwischenzeit kryokonserviert wurde, wieder implantiert werden. Im CT werden auch posttraumatische Defektbildungen sichtbar (e). Intraoperatives Bild einer Impressionsfraktur mit Kompression des Sinus sagittalis superior und Z. n. Hebung und Fixierung der Fraktur (f). Quelle: Mit freundlicher Genehmigung des Instituts für Klinische Radiologie der Medizinischen Fakultät und des Universitätsklinikums Münster (Direktor: Univ.-Prof. Dr. med. Walter Heindel).

- Tab. 2 Klassifikation nach dem GCS-Summenscore.

\begin{tabular}{|l|c|l|}
\hline Kategorie & GCS (Punkte) & Frankfurter GCS (Punkte) \\
\hline leichtes SHT & $13-15$ & $17-19$ \\
\hline mittelschweres SHT & $9-12$ & $12-16$ \\
\hline schweres SHT & $<8$ & $<11$ \\
\hline
\end{tabular}

die eingeschränkte verbale Antwortfähigkeit der Kinder moduliert wurde und als Frankfurter GCS (F-GCS) bezeichnet wird ( $\triangleright$ Tab. 1 und 2 ) [8].

\section{Symptomatik}

Neben Bewusstseinsstörungen können auch Symptome aus dem vegetativen Bereich auftreten wie Blässe, Übelkeit, Schwindel, Kopfschmerzen. Bei schweren Verletzungen treten u. U. fokal-neurologische Defizite wie Gefühlsstörungen und Lähmungen hinzu, bei schwersten Traumata auch relevante Atem- und Kreislauffunktionsstörungen.

\section{Verletzungsmuster}

Durch eine äußere Gewalteinwirkung auf den Kopf kann es zu unterschiedlichen Verletzungsmustern kommen: Weichteilverletzungen, die sehr stark bluten und somit das Kind gefährden können. Des Weiteren kann es zu einzelnen oder mehreren Schädelfrakturen kommen 

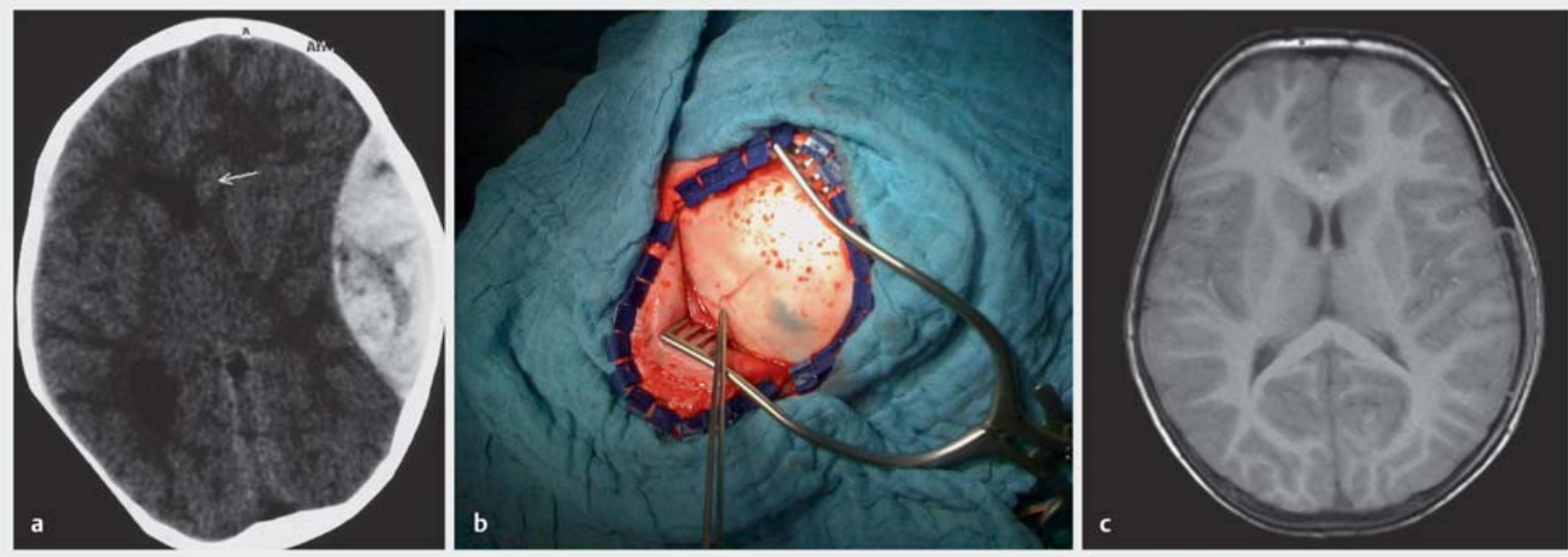

- Abb. 2 Fallbeispiel Epiduralhämatom: 3-jähriger Junge, der auf der Treppe gestürzt ist. Unmittelbar nach dem Unfall lag der GCS bei 15 (sog. „freies Intervall“), verschlechterte sich aber rasch präoperativ auf einen GCS von 5. Im Schädel-CT zeigte sich ein ausgedehntes Epiduralhämatom mit Mittellinienverlagerung (a). Intraoperativ ist häufig eine Frakturlinie oberhalb des Hämatoms zu erkennen sowie durch den Knochen bereits das Hämatom durchschimmernd (b). Ein postoperatives MRT zeigte eine vollständige Entfernung des Epiduralhämatoms (c). Quelle: Mit freundlicher Genehmigung des Instituts für Klinische Radiologie der Medizinischen Fakultät und des Universitätsklinikums Münster (Direktor: Univ.-Prof. Dr. med. Walter Heindel).

$(\triangleright$ Abb. 1 a,f), die je nach Ausmaß operativ behandelt werden müssen, insbesondere, wenn es gleichzeitig zur Zerreißung der harten Hirnhaut mit dem Risiko einer Liquorrhö kommt. Dieses Phänomen erhöht die Infektionsgefahr. Durch Ruptur von Gefäßen kann es zur lokalen Mangeldurchblutung oder zu diversen Blutungen intrakraniell kommen:

- epidural (zwischen Schädelknochen und Dura mater $\rightarrow$ Letalität bis $70 \%$; $\triangleright$ Abb. 2),

- subdural (zwischen Dura mater und Gehirn $\rightarrow$ Letalität bis $50 \%$; Abb. 1 b),

- subarachnoidal (zwischen Spinngewebshaut und Gehirn, an der Hirnoberfläche) intrazerebral (im Hirngewebe; - Abb. 1 d).

\section{Merke}

Bei Nachweis eines Schädel-Hirn-Traumas müssen Neurochirurgen rasch hinzugezogen werden, um im Falle einer Schädelfraktur, traumatischen Hirnblutung oder Hirnschwellung über die Notwendigkeit einer Operation zu entscheiden.

\section{Diagnostik}

\section{Die Wahl der Klinik}

Generell sind Indikationen zur stationären Einweisung Bewusstseinstrübung, neurologische Ausfälle, epileptische Anfälle, äußere Verletzungszeichen, rezidivierendes Erbrechen, schwerer Traumamechanismus, schwerwiegende Vorerkrankungen, Gerinnungsstörungen etc. Schlussendlich richtet sich die Wahl der Klinik nach ihrer Entfer- nung und Ausstattung. Im Falle eines schweren SHT mit GCS $<9$, zunehmender Vigilanzminderung und neurologischen Defiziten sollte eine Klinik mit der Möglichkeit einer sofortigen neurochirurgischen Versorgung sowie Expertise in kinderintensivmedizischer Betreuung angefahren werden [8].

\section{Anamnese}

Zum standardisierten Vorgehen bei allen Schädel-HirnVerletzten gehört eine genaue Anamneseerhebung am Unfallort mit Erfassung

- des Unfallgeschehens (Hochrasanztrauma?, Sturz aus großer Höhe?),

- des zeitlichen Ablaufs,

- der beobachteten Symptome.

\section{Klinische Beurteilung}

Initial sollte eine rasche klinische Beurteilung mit Augenmerk auf die folgenden Aspekte gerichtet werden:

- neurologischer Status

- Einschätzung nach (Frankfurter) GCS

- Nachweis äußerer Verletzungszeichen

- Hinweise auf epileptischen Anfall (Einnässen, Zungenbiss?)

Wesentlich ist die Erfassung von direkten oder indirekten Zeichen für ein schweres SHT mit hohem Risiko für intrakranielle Verletzungen (Infobox 1). Je nach Anamnese muss auch - insbesondere bei Säuglingen - die Möglichkeit von nicht akzidentellen Traumfolgen, z. B. im Sinne eines Schütteltraumas, gedacht werden (Infobox 2,

- Abb. 3). 

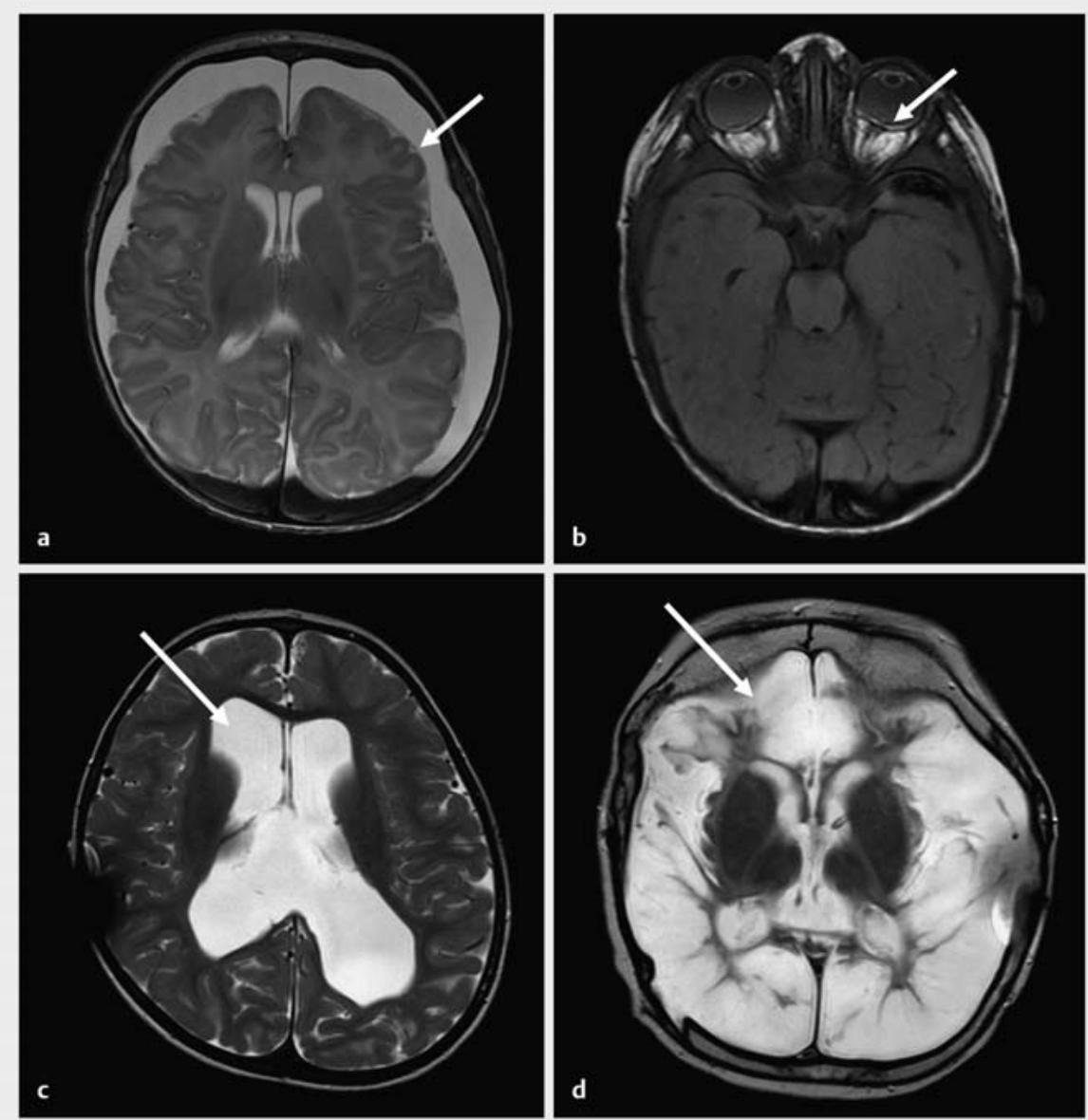

- Abb. 3 Fallbeispiel Schütteltrauma: 1,5 Monate alter männlicher Säugling mit V.a. Schütteltrauma. Nachweis ausgedehnter bilateraler Subduralhämatome (MRT T2ax, a), sowie retinaler Blutungen bds. (MRT T1ax, b). Im Langzeitverlauf Entwicklung eines posthämorrhagischen Hydrozephalus (MRT T2ax, c). Langzeitfolgen eines schweren Schütteltraumas mit ausgedehnten Parenchymdefekten des Großhirns (MRT T2ax, d). Quelle: Mit freundlicher Genehmigung des Instituts für Klinische Radiologie der Medizinischen Fakultät und des Universitätsklinikums Münster (Direktor: Univ.-Prof. Dr. med. Walter Heindel).

\section{INFOBOX 1}

Praxistipp: direkte und indirekte Verletzungszeichen

Direkte Zeichen:

- Galeahämatom

- Monokel- oder Brillenhämatom

- klinisch tastbare Kalottenfraktur

- Impressionsfraktur

- Rhino- oder Otoliquorrhö

- neurologisches Defizit

Indirekte Zeichen:

- initialer Bewusstseinsverlust

- Amnesie zum Unfallhergang

- Kopfschmerzen

- rezidivierendes Erbrechen

- Lethargie, Blässe u.a. vegetative Zeichen

\section{INFOBOX 2}

Hintergrundwissen: Schütteltrauma („Shaken Baby Syndrome [SBS]“)

Hinweise für nicht akzidentelle Traumafolgen

(Schütteltrauma) [4]:

- klinisch Zeichen einer schweren Hirnschädigung

- Subduralblutungen (bilateral, ggf. mehrzeitig)

- retinale Blutungen (meist bilateral)

- ggf. Rippenfrakturen, metaphysäre Frakturen

- fehlende, indadäquate oder inkonsistente Unfallanamnese

Abhängig von der initialen Einschätzung des Schweregrads des Traumas unter Berücksichtigung des Unfallmechanismus und dem Vorhandensein einer Bewusstseinsstörung (BWST) wird das weitere Vorgehen defi- 
niert. Insbesondere die niedrige Inzidenz von intrakraniellen Verletzungen rechtfertigt ein differenziertes Vorgehen und die Implementierung von Behandlungsalgorithmen, um so die Strahlenbelastung durch bildgebende Diagnostik als potenzielle Gefährdungsquelle für den Langzeitverlauf zu reduzieren [9].

Diagnostikalgorithmen sind für Kinder mit fehlender Bewusstseinsstörung und Patienten mit Nachweis einer Bewusstseinsstörung in $>$ Abb. $\mathbf{4}$ und $\mathbf{5}$ dargestellt.

Merke

Gerade bei Kindern unter 2 Jahren ist es für den Untersucher schwierig, Symptome eines Schädel-HirnTraumas wie auffällige Verhaltensänderungen, Schläfrigkeit, verminderte Spontanmotorik und verzögerte Reaktion zu erfassen. Daher kommt den Angaben der Bezugsperson hier eine große Bedeutung zu [10].

\section{Bildgebung}

Eine bildgebende Diagnostik sollte immer erfolgen, wenn eine Bewusstseinsstörung vorliegt und/oder sich in der klinischen Beurteilung Hinweise auf intrakranielle Verletzungsfolgen ergeben (Infobox 1).

Folgende Modalitäten stehen zur Verfügung:

- CT des Schädels: Methode der ersten Wahl zur Beurteilung akuter Verletzungen. Die Anwendung erfolgt nicht als Routinemaßnahme, sondern nur bei klinischem Verdacht auf das Vorliegen von intrakraniellen Verletzungen.

- Sonografie: durch die Fontanelle, sofern diese noch geöffnet ist, zur intrakraniellen Beurteilung sowie Sonografie der Kalotte zum Ausschluss von Schädelfrakturen.

- MRT des Schädels: weniger sinnvoll in der Akutdiagnostik bei V.a. schweres SHT (Untersuchungsdauer, ggf. Notwendigkeit einer Sedierung, Narkose), vertretbar bei milder oder fehlender neurologischer Symptomatik und einem Unfallmechanismus, der eine bildgebende Diagnostik erfordert; hoher Stellenwert zur Verlaufsbeobachtung und bei V.a. Scherverletzungen.

\section{Therapie}

\section{Merke}

Am Unfallort:

- Atem- und Kreislaufstabilisierung, Lagerung mit Stifneck (Halsstütze) auf einer Vakuummatratze (Cave: begleitende Wirbelsäulenverletzungen)

- offene Verletzungen mit sterilen Kompressen abdecken und nur kontrollierte Kompression dabei anwenden

- Intubation bei GCS < 9

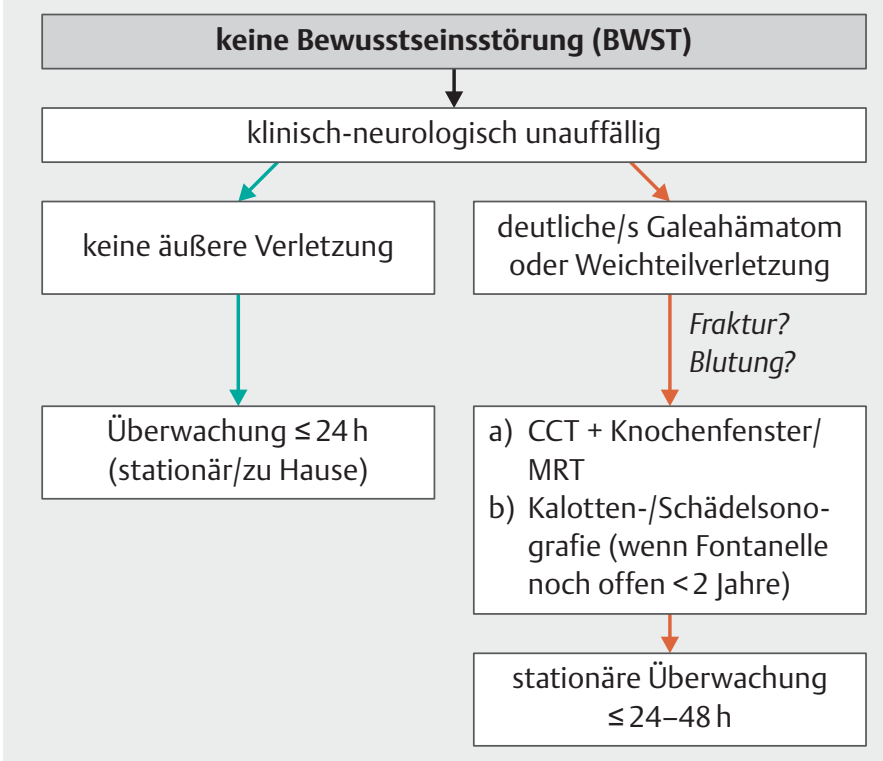

- Abb. 4 Algorithmus bei fehlender Bewusstseinstrübung. Algorithmus zur Herangehensweise bei Schädel-Hirn-traumatisierten Kindern ohne das Vorliegen einer Bewusstseinsstörung (BWST).

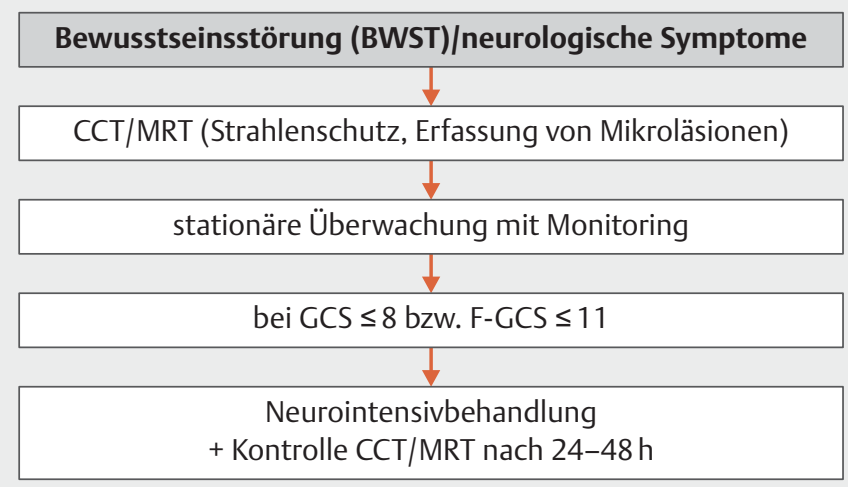

- Abb. 5 Algorithmus bei Vorhandensein einer Bewusstseinstrübung/ neurologischen Symptomen. Algorithmus zur Herangehensweise bei Schädel-Hirn-traumatisierten Kindern mit Vorliegen einer Bewusstseinsstörung (BWST) und/oder neurologischen Symptomen.

In der Klinik:

- Wundinspektion vor und Wundversorgung nach bildgebender Diagnostik

\section{Operative Therapie}

Den Zeitpunkt, die Reihenfolge und das Ausmaß einer operativen Therapie bei Kopfverletzungen muss ein fachübergreifendes Team unmittelbar nach erfolgter Primärdiagnostik festlegen. 
Dies erfolgt durch:

- Neurochirurgen,

- Unfallchirurgen,

- Hals-Nasen-Ohren-Ärzte,

- Augenärzte,

- Mund-Kiefer-Gesichts-Chirurgen

in Absprache und mit Unterstützung von:

- Anästhesisten und

- Kinderintensivmedizinern.

\section{Indikation zur invasiven Hirndruckmessung (ICP-Sonde)}

In Abhängigkeit von der Primärdiagnostik und der zu erwartenden Dauer einer Bewusstseinseinschränkung muss über die Anlage einer Hirndruckmesssonde (ICP-Sonde)/ externen Ventrikeldrainage entschieden werden. Insbesondere ermöglicht die invasive Hirndruckmessung die kontinuierliche Überwachung hinsichtlich progredienter Hirnschwellung, raumfordernder intrakranieller Hämatome sowie drohender Einklemmungen, sodass frühzeitig Gegenmaßnahmen ergriffen werden können. Ebenso kann über die Drainage Liquor abgelassen werden, was zu einer temporären Reduktion des Hirndruckes führt. Die Einführung von Leitlinien, die bei Patienten mit schwerem SHT die Versorgung mit einer ICP-Sonde fordern, hat zu einer Zunahme günstiger Verläufe bei SHTPatienten geführt $[11,12]$. Für Kinder gibt es diesbezüglich aktuell wenig Evidenz, dennoch besteht die Empfehlung zur Hirndruckmessung gemäß der Leitlinie bei Kindern mit schwerem bzw. mittelschwerem SHT, Nachweis von Traumafolgen in der Bildgebung sowie starker Bewusstseinsstörung und Notwendigkeit der längerfristigen invasiven Beatmung und Sedierung [8]. Dabei ist zu beachten, dass die Komplikationshäufigkeit bei Kindern höher ist als bei Erwachsenen. Dazu gehören Stichkanalblutungen, Liquorleckagen- und Dislokationen.

Säuglinge und Kleinstkinder mit noch offener Fontanelle können palpatorisch und sonografisch überwacht werden. Die Anlage einer ICP-Sonde ist in diesen Fällen primär nicht indiziert.

\section{Indikation zur Operation}

Einer notfallmäßigen operativen Versorgung über eine Kraniotomie bedürfen sowohl raumfordernde, intrakranielle Verletzungen, wie z.B. traumatische Blutungen (Epiduralhämatome, Subduralhämatome, intrakranielle Blutungen), als auch raumfordernde Impressionsfrakturen ( $\bullet$ Abb. 1). Neben dem bildgebenden Befund ist auch der klinische Befund entscheidend für die Stratifizierung der Notwendigkeit und Dringlichkeit der Operation.
Trotzdem ist die Prognose bei sofortiger chirurgischer Therapie bei dieser Hirnverletzung die beste ( Abb. 2).

Dringliche Indikation zur operativen Versorgung haben basale Frakturen mit Liquorrhö sowie Impressionsfrakturen ohne raumfordernde Komponente.

Bei Entwicklung eines ausgeprägten - meist sekundären - Hirnödems und konservativ nicht zu therapierenden erhöhten Hirndrücken kann die Durchführung einer Entlastungskraniektomie erforderlich sein. Deren Notwendigkeit ist jedoch insbesondere bei Kindern kritisch zu sehen.

\section{Konservative Therapie}

Konservative Therapie bei leichtgradigem Schädel-Hirn-Trauma

- stationäre Überwachung (siehe z. B. auch • Abb. 6, Ping-Pong-Fraktur)

- engmaschige neurologische Verlaufsuntersuchungen

- bei Wohlbefinden: Entlassung nach Hause, hierzu Absprache mit den Eltern und Sensibilisierung der Eltern für Symptome, die auf Komplikationen des SHTs hinweisen

\section{INFOBOX 3}

\section{Praxistipp}

Monitoring bei Neurointensivbehandlung (GCS $\leq 8$

bzw. F-GCS $\leq 11$ nach AWMF-Leitlinie [8]

- Hirndruckmessung (ICP), kontinuierlich

- arterielle Druckmessung (MAD), kontinuierlich

- zentralvenöse Druckmessung (ZVD), möglichst kontinuierlich

- Messung der arteriellen Sauerstoffsättigung (Pulsoxymetrie) und des endtidalen $\mathrm{CO}_{2}$, kontinuierlich

- EKG-Ableitung, kontinuierlich

- genaue Flüssigkeitsbilanzierung

- engmaschige Überwachung der Körpertemperatur

- Laborkontrollen

- wenn möglich wiederholt Elektroenzephalografie (EEG), Akustische Evozierte Potenziale (AEP), Sensible Evozierte Potenziale (SEP)

- wenn möglich transkranielle Doppler-Sonografie

- neurologische und allgemeinpädiatrische Untersuchung (alle 4-6 Stunden, mindestens $1 \times$ täglich)
Merke

Epidurale Hämatome können sich trotz zunächst guten Befindens innerhalb von wenigen Stunden (sog. freies Intervall) zur tödlichen Verletzung entwickeln. 

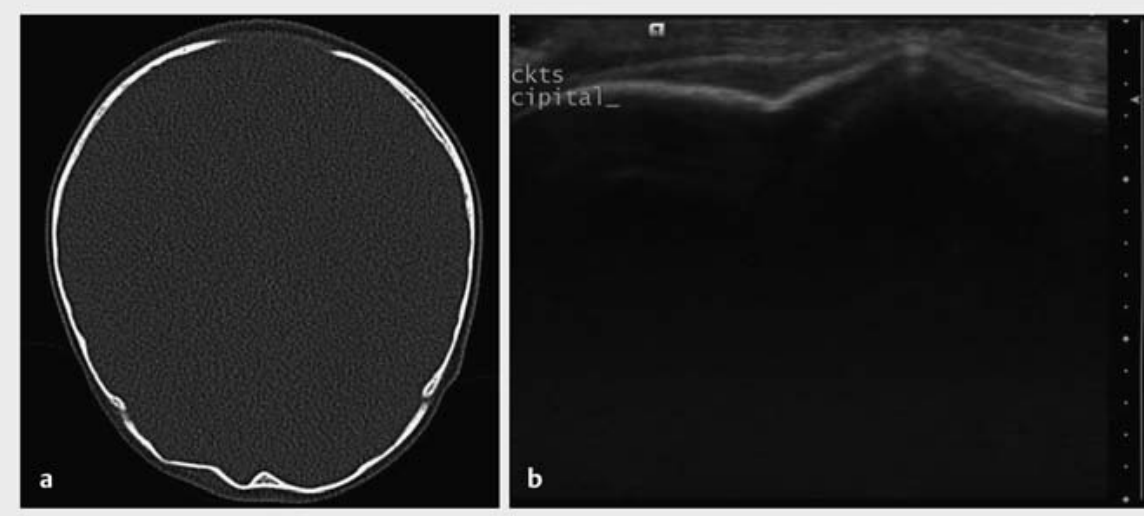

- Abb. 6 Fallbeispiel Ping-Pong-Fraktur: Ein 9 Monate altes Mädchen mit Z.n. Sturz auf den Hinterkopf zeigte klinisch eine tastbare Impression. Im CT stellt sich eine Impressionsfraktur rechts okzipital dar (a). In der Sonografie des Schädels lässt sich diese PingPong-Fraktur (Schädel ist wie ein eingedrückter Tischtennisball verformt) gut abgrenzen (b). Die meisten heilen konservativ gut aus, eine Operation ist nur noch bei neurologischen Symptomen oder höhergradiger Kompression des Hirnparenchyms indiziert. Quelle: Mit freundlicher Genehmigung des Instituts für Klinische Radiologie der Medizinischen Fakultät und des Universitätsklinikums Münster (Direktor: Univ.-Prof. Dr. med. Walter Heindel).

Konservative Therapie bei mittel- oder schwergradigem Schädel-Hirn-Trauma

Zur (Neuro-)Intensivbehandlung auf einer Kinderintensivstation gehören nach einem SHT:

- die Lagerung mit 20-30 Oberkörperhoch- und Kopfmittelstellung,

- das "minimal handling“ bei Pflegemaßnahmen, d.h. nur dringend erforderliche Maßnahmen,

- die Flüssigkeits-/Volumenzufuhr mit dem Ziel eines zentralen Venendruckes (ZVD) von 5-8 $\mathrm{mmHg}$,

- die Beatmung mit milder Hyperventilation und einem arteriellen $\mathrm{pCO}_{2}$ von $35-40 \mathrm{mmHg}$,

- die Analgosedierung mit z.B. Midazolam und einem Fentanylpräparat,

- das Einsetzen von Antikonvulsiva zur Krampfprophylaxe mit z. B. Phenobarbital.

\section{Rehabilitation}

Die Behandlung nach der akuten Phase einer SchädelHirn-Verletzung wird auch bei den jungen Patienten nach dem Phasenmodell der Bundesarbeitsgemeinschaft (BAR) für Rehabilitation durchgeführt ( $\bullet$ Tab. 3).

Die Indikation zur neurologischen Rehabilitation eines Kindes oder Jugendlichen nach akuter Schädigung des Nervensystems besteht, wenn gegen Ende der Akutbehandlung fortbestehende Störungen körperlicher, geistiger oder seelischer Funktionen bestehen bzw. anzunehmen sind [13] (siehe auch $>$ Abb. 7).

Insbesondere posttraumatische neuropsychiatrische Störungen haben ein verzögertes Auftreten, häufig erst nach 1,5-2 Jahren. Diese können auch nach leichten SHT entstehen. Eltern und Bezugspersonen sollten deshalb schon im Vorfeld dafür sensibilisiert werden und langfristig mit einem Netzwerk in Kontakt stehen. Hier bietet sich der Bundesverband Kinderneurologie-Hilfe e.V. an, der sich seit über 30 Jahren bundesweit für Kinder mit erworbenen Hirnschädigungen nach Kopfverletzungen einsetzt.

- Tab. 3 Phasen der Rehabilitation.

\begin{tabular}{|c|c|c|}
\hline Rehabilitation & Frührehabilitation & Akutklinik \\
\hline $\begin{array}{l}\text { Phase D: } \\
\text { Patienten, die weitestgehend selbstständig } \\
\text { sind }\end{array}$ & $\begin{array}{l}\text { Phase B: } \\
\text { überwachungspflichtige Patienten mit schwe- } \\
\text { ren Störungen des Bewusstseins, der Wahr- } \\
\text { nehmung und Kommunikation } \\
\text { Phase C: } \\
\text { Patienten, die noch einen hohen pflegerischen } \\
\text { Hilfsmittel- und Betreuungsbedarf haben }\end{array}$ & $\begin{array}{l}\text { Phase A: } \\
\text { Akutphase (Operationen, Intensivmedizin) }\end{array}$ \\
\hline
\end{tabular}




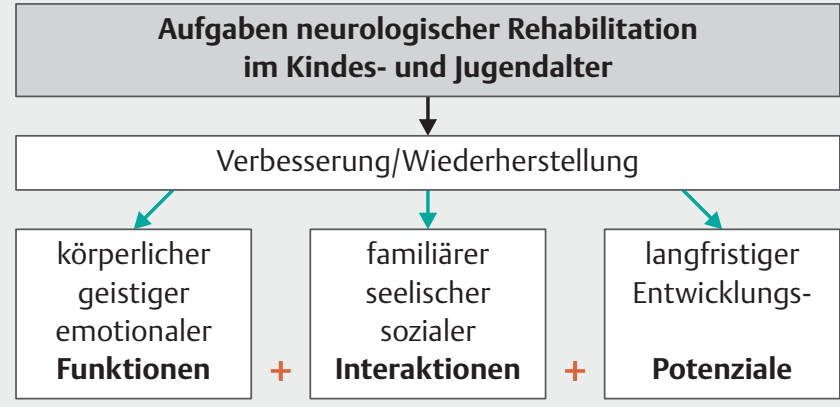

- Abb. 7 Flussdiagramm - Aufgaben neurologischer Rehabilitation im Kindes- und Jugendalter.

\section{INFOBOX 4}

Info

Kinder- und Jugendrehabilitationskliniken mit Expertise in der Rehabilitation von SHT-Patienten (Auswahl)

- Helios Klinik Hohenstücken, 14772 Brandenburg

- St. Mauritius Therapieklinik, 40670 Meerbusch

- Helios Klinik Geesthacht, 21502 Geesthacht

- Helios Klinik Hattingen, 45527 Hattingen

- Schön Klinik Vogtareuth, 83569 Vogtareuth

Kontakthinweis

Bundesverband Kinderneurologie-Hilfe e. V.

E-Mail: info@Kinderneurologiehilfe.de

Internet: www.kinderneurologiehilfe.de

\section{Nachsorge}

Posttraumatische Kontrolluntersuchungen dienen der Aktualisierung des Ausmaßes der traumatischen Schädigung. Sie sind Grundlage für die Begründung von Neuverordnungen von Therapien und Hilfsmitteln. Die Prognoseeinschätzung beruft sich ebenfalls auf die Befunde der Nachuntersuchungen.

\section{Merke}

Nachuntersuchungen bei Z.n. SHT

- klinisch-neurologische Untersuchung

- MRT

- EEG und evtl. evozierte Potenziale

- standardisierte Testverfahren (somatische, kognitive und psychologische)

\section{INFOBOX 5}

\section{Hintergrundwissen}

Posttraumatische Epilepsie wird unterschätzt

- $10,9 \%$ mit einem leichten SHT ^ GCS 13-15 (Commotio) erleiden mindestens einen epileptischen Anfall

- 5-21\% aller Kinder erleiden posttraumatisch einen Anfall

- 32-40\% davon haben wiederholte Anfälle

- Insgesamt ist das Risiko für die Kinder, nach einem SHT an Epilepsie zu leiden, $8 \times$ größer als bei der Normalbevölkerung [14]

\section{Prognose und Langzeitfolgen}

Der Langzeitverlauf nach einer Hirnverletzung bei Kindern ist sehr individuell und schlecht vorhersagbar. Die Kontrolluntersuchungen, insbesondere MRT-Aufnahmen, können eine vorsichtige Aussage über den weiteren Verlauf ermöglichen. So wird die Primärschädigung der sog. weißen Hirnsubstanz und auch ihre Weiterentwicklung in der Kernspintomografie sichtbar [15].

Die aktuelle Literatur ist bez. prognostischer Zahlen bei einem kindlichen SHT und dessen langfristigen Verlaufs nicht ergiebig. Aus dem Flyer des Registers für SHT-Erkrankungen erfährt man, dass Patienten mit mittelschwerem SHT in 77\% der Fälle nach 273 Tagen und in 83\% der Fälle nach 730 Tagen wieder die Schule besuchen [10].

In einer japanischen Studie aus dem Jahr 2017 wird angegeben, dass Kinder mit einem schweren SHT zu 56,6\% innerhalb eines Jahres sterben und 15\% ein gutes Befinden nach 10 Jahren zeigen [16].

Eine Metaanalyse aus den USA (2013) ergab Verhaltensstörungen von $50 \%$ bei den hirnverletzten Kindern [17]. Eine andere Arbeit aus Australien (2015) unterstützt die These der psychischen Spätfolgen [18].

Dennoch können Kinder mit schweren Hirnverletzungen nach entsprechend rascher Notfallversorgung und Rehabilitation eine gute Prognose aufweisen, da bei jungen Menschen das Gehirn eine erhöhte Flexibilität, Entwicklungsplastizität und Möglichkeit der Regeneration insbesondere von sprachlichen und intellektuellen Fähigkeiten aufweist [19]. 
INFOBOX 6

Hintergrundwissen

Mögliche Spätfolgen nach Schädel-Hirn-Trauma [20,

21].

- kognitive Leistungsminderungen

- Konzentrationsstörungen

- Aufmerksamkeitsstörungen

- soziale Anpassungsstörungen

- Entwicklungsverzögerung

- emotionale Defizite (Labilität, Apathie)

\section{Prävention}

Schädel-Hirn-Verletzungen lassen sich durch vorbeugende Schutzmaßnahmen zu über 90\% vermeiden [22].

Da Stürze aus der Höhe bei Säuglingen häufig zu schweren Verletzungen führen und vermeidbar sind, sollten insbesondere unerfahrene Eltern verstärkt darüber aufgeklärt werden. Normale Altersgegenstände stellen schon ein Risiko dar.

Zu Hause sollte durch kontinuierliche Aufsicht bei Sturzgefahr (Wickeltisch etc.) die Gefahr reduziert, wenn nicht ausgeschlossen werden. Auch Antirutschsocken oder feste Schuhe bedeuten dort eine Vorsorge. Hochbetten sind erst für Schulkinder geeignet und dann mit entsprechender Sicherheitsvorrichtung. Im Straßenverkehr sind altersgemäße Sitze (Auto und Fahrrad) zu benutzen. Beim Fahrradfahren und bei sportlichen Aktivitäten ist ein entsprechender Schutzhelm immer zu tragen.

\section{Fazit}

- Die Anzahl schwerer Schädel-Hirn-Traumata hat wegen gezielter Präventionsmaßnahmen (Helm, Kindersitz, etc.) abgenommen, nicht aber die Zahl gewaltbedingter Schädelverletzungen.

- Das Schädel-CT ist die Methode der ersten Wahl in der Notfalldiagnostik bei V.a. das Vorliegen einer intrakraniellen Verletzung, bei Säuglingen und Kleinkindern die Sonografie.

- Das MRT ist die Methode der ersten Wahl zur geplanten/differenzierten Verlaufskontrolle und Einschätzung der Prognose nach Schädel-Hirn-Verletzung.

- Bei V.a. mittelschweres oder schweres SHT sollte möglichst direkt eine Klinik mit neurochirurgischer Expertise ausgewählt werden.

- Eine Hirndruckmesssonde sollte bei Kindern wegen erhöhter Komplikationsrate mit Zurückhaltung gelegt werden. Sie ist jedoch indiziert bei verschlossener Fontanelle, wenn mit einer längeren Beatmungszeit zu rechnen ist oder wenn in der initialen Bildgebung bereits schwere Verletzungsfolgen oder eine Hirnödembildung zu sehen sind.
- Eine Beurteilung über Langzeitfolgen nach SHT bei Kindern ist frühestens nach 2 Jahren möglich.

Interessenkonflikt

Es bestehen keine Interessenkonflikte oder Veröffentlichungen in anderen Zeitschriften oder Medien.

\section{Autorinnen/Autoren}

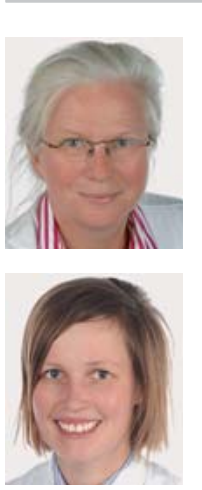

\section{Angela Brentrup}

Dr. med., Leiterin der Pädiatrischen Neurochirurgie, Klinik für Neurochirurgie, Universitätsklinikum Münster

\section{Stephanie Schipmann}

Dr. med., Assistenzärztin, wissenschaftliche Mitarbeiterin,, Klinik für Neurochirurgie, Universitätsklinikum Münster

Korrespondenzadresse

\section{Dr. med. Angela Brentrup}

Klinik für Neurochirurgie - Pädiatrische Neurochirurgie

Universitätsklinikum Münster

Albert-Schweitzer-Campus A1

48149 Münster

Tel.: 0251/83-47474

Fax: 0251/83-47479

angela.brentrup@ukmuenster.de

Literatur

[1] Firsching R, Rickels E, Mauer UM et al. Schädel-Hirn-Trauma im Erwachsenenalter. AWMF-Leitlinien-Register 2007. Im Internet: https://www.awmf.org/leitlinien/detail/l/008-001.html; Stand: 01.06 .2018

[2] Rickels E, von Wild K, Wenzlaff P. Head injury in Germany: a population-based prospective study on epidemiology, causes, treatment and outcome of all degrees of head-injury severity in two distinct areas. Brain Inj 2010; 24: 1491-1504. doi:10.3109/02699052.2010.498006

[3] Ellsäßer G. Unfälle, Gewalt, Selbstverletzung bei Kindern und Jugendlichen 2017. Ergebnisse der amtlichen Statistik zum Verletzungsgeschehen 2014. Statistisches Bundesamt; 2017. Im Internet: https://www.destatis.de/DE/Publikationen/Thematisch/Gesundheit/Gesundheitszustand/UnfaelleGewaltKinder5230001149004.pdf?__blob=publicationFile; Stand: 01.06 .2018

[4] Matschke J, Herrmann B, Sperhake J et al. Shaken baby syndrome: a common variant of non-accidental head injury in infants. Dtsch Arztebl Int 2009; 106: 211-217. doi:10.3238/ arztebl.2009.0211

[5] Greve MW, Zink BJ. Pathophysiology of traumatic brain injury. Mt Sinai J Med 2009; 76: 97-104. doi:10.1002/msj.20104

[6] Ibrahim NG, Wood J, Margulies SS et al. Influence of age and fall type on head injuries in infants and toddlers. Int J Dev Neurosci 2012; 30: 201-206. doi:10.1016/j.ijdevneu.2011.10.007 
[7] Ellsäßer G, Diepgen TL. Epidemiologische Analyse von Sturzunfällen im Kindesalter ( $<15$ Jahre) in Deutschland. Erarbeitet im Auftrag des Fördervereins der Bundesvereinigung für Gesundheit e.V., handelnd für die Bundesarbeitsgemeinschaft Kindersicherheit, Bonn, 2014. Im Internet: http://www.kinderumweltgesundheit.de/index2/pdf/themen/Unfaelle/expertise_sturzunfaelle.pdf; Stand: 01.06.2018

[8] Gesellschaft für Neonatologie und pädiatrische Intensivmedizin e.V. Das Schädel-Hirn-Trauma im Kindesalter. AWMFLeitlinien-Register 2011. Im Internet: https://www.awmf.org/ leitlinien/detail/I/024-018.html; Stand: 01.06.2018

[9] Goldberg J, McClaine RJ, Cook B et al. Use of a mild traumatic brain injury guideline to reduce inpatient hospital imaging and charges. J Pediatr Surg 2011; 46: 1777-1783. doi:10.1016/j. jpedsurg.2011.02.052

[10] Schnadower D, Vazquez H, Lee J et al. Controversies in the evaluation and management of minor blunt head trauma in children. Curr Opin Pediatr 2007; 19: 258-264. doi:10.1097| MOP.0b013e3281084e85

[11] Fakhry SM, Trask AL, Waller MA et al. Management of brain-injured patients by an evidence-based medicine protocol improves outcomes and decreases hospital charges. J Trauma 2004; 56: 492-499

[12] Palmer S, Bader MK, Qureshi A et al. The impact on outcomes in a community hospital setting of using the AANS traumatic brain injury guidelines. Americans Associations for Neurologic Surgeons. J Trauma 2001; 50: 657-664

[13] Deppe W, Köhler DW, Lütjen S, Spranger M, Voss A, Wright M. Leitlinie Neurologische Rehabilitations im Kindes- und Jugendalter. Fachgesellschaft Rehabilitation in der Kinder- und Jugendmedizin. Im Internet: https://slidex.tips/download/fachgesellschaft-rehabilitation-in-der-kinder-und-jugendmedizinleitlinie-neurol; Stand: 18.06.2018

[14] Keret A, Bennett-Back O, Rosenthal G et al. Posttraumatic epilepsy: long-term follow-up of children with mild traumatic brain injury. J Neurosurg Pediatr 2017; 20: 64-70. doi:10.3171/2017.2.PEDS16585

[15] Königs M, Pouwels PJ, Ernest van Heurn LW et al. Relevance of neuroimaging for neurocognitive and behavioral outcome after pediatric traumatic brain injury. Brain Imaging Behav 2018; 12: 29-43. doi:10.1007/s11682-017-9673-3

[16] Araki T, Yokota H, Morita A. Pediatric traumatic brain injury: characteristic features, diagnosis, and management. Neurol Med Chir (Tokyo) 2017; 57: 82-93. doi:10.2176/nmc. ra.2016-0191

[17] Li L, Liu J. The effect of pediatric traumatic brain injury on behavioral outcomes: a systematic review. Dev Med Child Neurol 2013; 55: 37-45. doi:10.1111/j.1469-8749.2012.04414.x

[18] Ryan NP, van Bijnen L, Catroppa C et al. Longitudinal outcome and recovery of social problems after pediatric traumatic brain injury (TBI): Contribution of brain insult and family environment. Int J Dev Neurosci 2016; 49: 23-30. doi:10.1016/j.ijdevneu.2015.12.004

[19] Classen J, Liepert J, Wise SP et al. Rapid plasticity of human cortical movement representation induced by practice. I Neurophysiol 1998; 79: 1117-1123. doi:10.1152/jn.1998.79.2. 1117

[20] Campbell CG, Kuehn SM, Richards PM et al. Medical and cognitive outcome in children with traumatic brain injury. Can J Neurol Sci 2004; 31: 213-219

[21] Kapapa T, Pfister U, König $\mathrm{K}$ et al. Head trauma in children, part 3: clinical and psychosocial outcome after head trauma in children. J Child Neurol 2010; 25: 409-422. doi:10.1177/ 0883073809340697

[22] Saß AC, Poethko-Müller C, Rommel et al. Das Unfallgeschehen im Kindes- und Jugendalter - Aktuelle Prävalenzen, Determinanten und Zeitvergleich. Bundesgesundheitsblatt Gesundheitsforschung Gesundheitsschutz 2014; 57: 789-797. doi:10.1007/s00103-014-1977-5

\section{Bibliografie}

DOI https://doi.org/10.1055/a-0623-5848

OP-JOURNAL 2018; 34: 232-242 @ Georg Thieme Verlag KG Stuttgart · New York ISSN 0178-1715 